\title{
Installation for Adiabatic Demagnetization Experiments at the National Bureau of Standards
}

\author{
D. de Klerk ${ }^{1}$ and R. P. Hudson
}

\begin{abstract}
A description is given of the National Bureau of Standards equipment for the production and measurement of very low temperatures by the method of adiabatic demagnetization. The construction of the cryostat and that of the mutual-inductance bridge are described in detail and the relative advantages of iron-cored electromagnets and iron-free solenoids are discussed.
\end{abstract}

\section{Introduction}

In recent years, with the rapid increase in the number of low-temperature laboratories throughout the world, there has been a coincident growth in the number of installations for the production and measurement of very low temperatures by the magnetic method. In the period from July 1951 to July 1952, an adiabatic demagnetization apparatus was set up at the National Bureau of Standards. A detailed description of this installation is given. It was built with the special purpose of making accurate determinations of absolute temperatures in the region between $1^{\circ}$ and $0.001^{\circ} \mathrm{K}$, but the construction is such that other investigations in the region below $1^{\circ} \mathrm{K}$ can also be performed with it.

\section{General Description}

The principle of cooling by adiabatic demagnetization was enunciated in $1926[1]^{2}$ and first applied successfully 7 years later [2]. A detailed description of the physical principles involved would not be appropriate here; for this the reader is referred to the literature [3]. Briefly, however, use is made of the fact that certain salts retain their paramagnetic properties down to temperatures in the region of $10^{-2} \circ \mathrm{K}$ and possess, at liquid-helium temperatures, a large entropy by virtue of their almost free electronic magnetic moments. This entropy may be removed for the greater part by isothermal magnetization at about $1^{\circ} \mathrm{K}$ in readily obtainable fields. A subsequent adiabatic removal of the field will result in a fall in the temperature of the salt, until the small internal interaction forces in the crystal bring about the same degree of order as does the strong magnetic field at $1^{\circ} \mathrm{K}$. It is self-evident that the final temperature is the lower, the stronger is the magnetic field and the lower the starting temperature. In fact, for most of the salts used in the adiabatic demagnetization process the entropy removed in the isothermal magnetization is a function of $H / T$.

Thus one needs a suitable paramagnetic salt in a cryostat with liquid helium of as low a temperature as possible, and mounted in such a way that it can be magnetized in heat contact with the liquid

1 Present address: University of Leiden, Netherlands.

2 Figures in brackets indicate the literature references at the end of this paper. (isothermally) and demagnetized under thermal insulation (adiabatically).

The cryostat is located between the poles of an electromagnet or along the axis of a high-power solenoid. With an iron magnet one can obtain fields of the order of 20,000 oersteds, making use of the high $B$ value of the iron. If much higher fields are required (up to 100,000 oersteds), the contribution of iron is relatively small and high-power iron-free solenoids are used. The energy consumption in the case of an iron magnet is of the order of $25 \mathrm{kw}$; the above-mentioned solenoids require about $1,000 \mathrm{kw}$.

In order to obtain a low starting temperature for the demagnetization process, the liquid helium is evaporated under reduced pressure. This is accomplished by means of a large-capacity mechanical vacuum pump operating through a large-diameter pumping line. A suitable diffusion pump inserted in the system may bring about a further reduction in the vapor pressure. (As mentioned above, the entropy removal in the isothermal magnetization is a function of $H / T$, and it is a more economical process to achieve a very low $T$ rather than an enormously high $H$ ).

The heat contact between the salt and the liquidhelium bath is made and broken with the help of exchange gas: The sample is mounted in a vacuum space, and during the isothermal magnetization the space is filled with helium gas at a pressure of $10^{-3}$ to $10^{-2} \mathrm{~mm} \mathrm{Hg}$. Thereafter the gas is pumped off with a diffusion pump and then the field is removed. As the specimen is now thermally isolated from the surrounding liquid, the demagnetization is isentropic and the temperature of the salt falls.

The final temperature, which is well below $1^{\circ} \mathrm{K}$, cannot be measured by conventional high-temperature methods, but indirect processes must be applied, often rather laborious ones [4], in which use is made of the magnetic properties of the salt, such as the static susceptibility $\chi$, the real and imaginary parts of the dynamic susceptibility, $\chi^{\prime}$ and $\chi^{\prime \prime}$, or, in the case that hysteresis effects occur, the residual magnetic moment $\Sigma$. These quantities, the so-called "thermometric parameters", are usually determined by an induction bridge method. Both self- and mutual-inductance bridges are in use, and the measurement can be performed ballistically as well as with alternating current. An a-c method has the advantage that many measurements can be taken in 
a short time and with high precision, but ballistic methods become particularly useful at the very lowest temperatures where relaxation and hysteresis effects appear, since here an alternating magnetic field may cause an appreciable heat supply to the salt.

If an iron-cored magnet is employed, provision must be made for the separation of the cryostat and electromagnet immediately after the demagnetization because the iron may have a considerable influence on the setting of the induction bridge for the temperature determination. If the magnet is relatively small, it is possible to have a cryostat in a fixed position and to mount the magnet on wheels or an elevating mechanism, but for a very heavy magnet this is impossible, and the cryostat with its pumping lines must be movable. If a solenoid magnet is used, its influence on the induction coils is much smaller, but even in this case the coupling between the measuring coils and the coaxial windings of the large solenoid produces undesirable effects unless special precautions are adopted in the construction of the measuring coils [5].

It is often desirable to study the magnetic properties of the salt as a function of a magnetic field, and if relatively low fields (up to about 500 oersteds) are sufficient, it is advantageous, for the above-mentioned reasons, not to use the big electromagnet or solenoid itself but to construct a separate small iron-free coil magnet. The influence of the coupling of this magnet with the measuring coils can be decreased drastically by mounting them mutually perpendicular.

In some cases even the earth's magnetic field has a noticeable influence on the magnetic properties of the salt, and then special earth-field compensation coils must be installed.

The components of the new NBS demagnetization equipment are described separately in the following sections.

\section{Electromagnet}

The electromagnet is a rotatable, adjustableheight model [6]. The pole gap, which must be as small as possible, was determined by the amount of space necessary to accommodate a salt specimen of about 1-in. diameter surrounded by a vacuum chamber and two glass Dewar vessels, the inner one of which contains liquid helium and the outer liquid nitrogen. The gap is $2 \frac{1}{4} \mathrm{in}$. and the pole face $5 \frac{3 / 4}{\mathrm{in}}$. Designed to be operated up to a level of $125 \mathrm{kw}$, the magnet is being used at present at $25 \mathrm{kw}$, the rating of the available $d-c$ generator. The loss in magnetic field is not great, however, owing to the rapid saturation that sets in at about $20 \mathrm{kw}$. At $25 \mathrm{kw}$ the field is 23,000 oersteds; at $125 \mathrm{kw}$ it is 26,000 oersteds. The magnet was calibrated, using a Rawson "gaussmeter", checked in turn in fields stabilized by proton resonance, through the kind cooperation of Dr. Hipple. The accuracy of this instrument is about one-half percent, and within this limit the field was found to be uniform out to within $1 \frac{1}{2} \mathrm{i}$ in. of the edge of the pole face.
The magnet is energized by a four-unit a-c-d-c synchronous motor-generator set mounted in the basement of the Bureau's Cryogenics Laboratory. This method has the advantage that the complete control of the magnet current, and the action of the protection devices (against, for instance, failure of cooling supply, overload, surge on current-break, etc.) can take place through the exciter field. The generator is started with a motor-control panel, and the current is adjusted at a separate exciter-control panel. Both panels are placed in the magnet room. The current is measured accurately by means of a millivoltmeter and a series of $50-\mathrm{mv}$ shunts.

Several automatic control circuits have been developed for the elimination of slow drifts in the magnet current [7], but in the present case manual adjustment proved to be satisfactory. A more serious source of trouble may be the ripple in the magnet current, which has the commutator frequency. If the ripple in the field is large, it will cause relaxation heating in the paramagnetic salt during the evacuation of the exchange gas, such that, by the time that the field is removed, the starting temperature of the demagnetization is much higher than that of the liquid-helium bath. For any frequency above a few hundred cycles per second the solid mass of iron in the electromagnet becomes ineffective, and the only choking effect is due to the self-inductance of the coils, which is generally quite small. But, on the other hand, in the contribution of the ripple current to the magnetic field the iron is ineffective as well, and this is one reason why in most iron magnets the influence of the ripple voltage of the generator on the field is negligible. Another is, of course, that the windings are generally quite far removed from the pole fgap and thel direct effect of the current is sman.

The ripple voltage, with a load current of $100 \mathrm{amp}$ d-c, was $2 \mathrm{v}$ at a frequency of $2,400 \mathrm{c} / \mathrm{s}$ (commutator ripple). From the values of the self-inductance at $\nu=2,400$ and $\nu=0$ and from the initial slope of the calibration curve of the magnet, it was estimated that this ripple voltage might cause a ripple in the field of not over 0.04 oersted. No ripple in the field could be detected by means of a search coil connected to a cathode-ray oscilloscope. It should have been possible in this way to detect 0.1 oersted at this frequency. (With respect to the ripple, an ironcored magnet has an advantage over an iron-free high-power solenoid. In the latter the ripple voltage is higher because a higher energy is used, and moreover the contribution of the ripple voltage to the field is larger because the windings are much closer to the field space than in the case of a Weiss-type magnet. Usually, special precautions must be taken to filter out the ripple current of a solenoid magnet.)

The magnet is cooled by circulating a low viscosity silicone oil through the coils and through a heat exchanger, the heat being carried off by means of tap water. The heat exchanger and the circulation pump are mounted in the basement just below the magnet, but the circulation can be started and stopped from the magnet room. 


\section{Movable Cryostat and Vacuum Installations}

The cryostat, consisting essentially of two coaxial Pyrex Dewar vessels, is mounted on the far end of a rotating wooden framework that pivots, by means of steel collars, on a vertical steel pillar. This framework carries the entire high-vacuum system and a 3-in. copper pumping line, which enters the side of a brass cryostat cap. The whole installation is shown in figure 1, and a vertical cross section of the cryostat is given in figure 2 .

The inner Dewar vessel (liquid helium) is fitted with its upper end in a brass ring held in place by sealing wax. The ring fits smoothly into the cryostat cap on the wooden arm and thus alines the Dewar vertically. A wired-on rubber sleeve provides vacuum tightness and helps support the weight of the cryostat. The outer vessel (liquid nitrogen) fits around the liquid-helium Dewar; it also is provided with a brass ring, which fits into a brass cap waxed to the outer

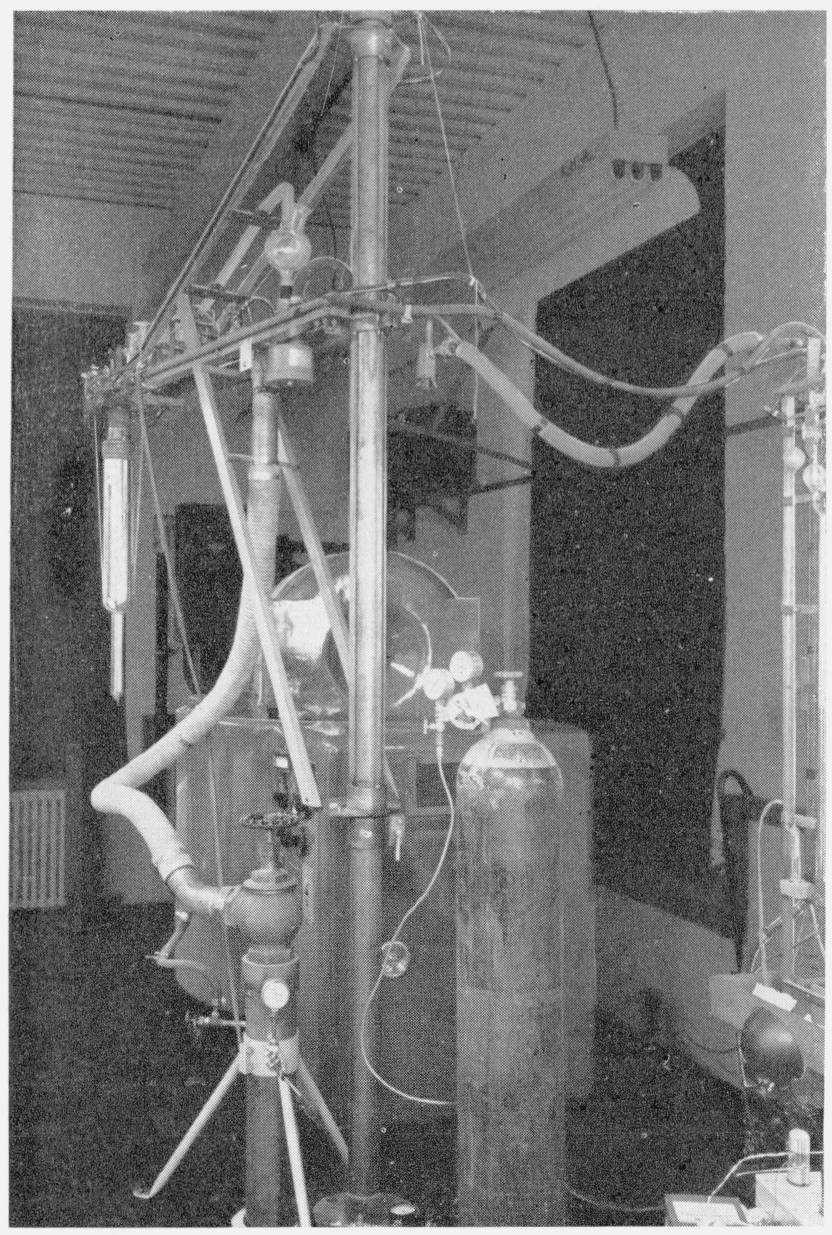

Figure 1. General view of the adiabatic demagnotization installation.

The Dewar-vessel assembly is suspended from the far end of the rotating arm, which carries the high-vacuum equipment. The electromagnet is in the background. wall of the helium Dewar. This ring is also a close fit in its cap, and with a rubber sleeve over this joint, too, the weight may be supported; for extra safety, however, a simple harness of string is provided.

The helium Dewar becomes single-walled about $20 \mathrm{~cm}$ from its upper end and about $10 \mathrm{~cm}$ below the nitrogen cap. If the nitrogen Dewar is kept well-
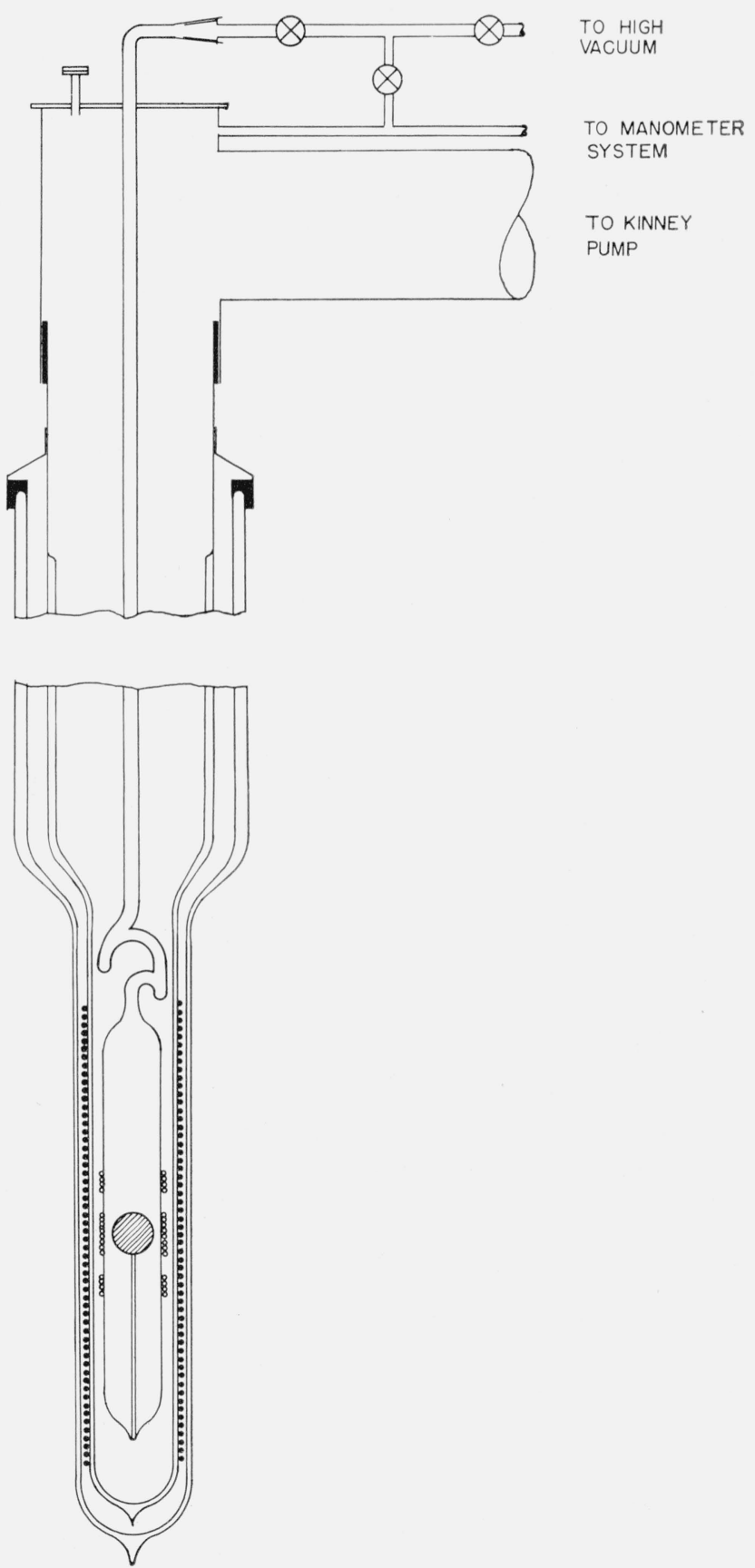

Figure 2. Cryostat.

The paramagnetic specimen is mounted within the vacuum case, on which is wound the secondary of the pickup mutual inductance. The primary of the latter is shown on the outside of the tail of the inner (liquid helium) Dewar vessel. 
filled, the rim of the helium vessel is at liquid-nitrogen temperature, and the heat leak to the liquid helium is smaller than if the rim were at room temperature. Hence the evaporation is less and experiments can be carried out for a longer time.

The two Dewar vessels are made with a narrow lower portion (the "tail") so that, although containing a large quantity of refrigerant, they may fit into a relatively small pole gap in the magnet. The tail of the liquid-helium Dewar is $33 \mathrm{~cm}$ long and $3.2-\mathrm{cm}$ inside diameter; it then widens to 7 -cm inside diameter for $43 \mathrm{~cm}$ up to the ring seal; the singlewalled section (20 cm long) is 8 -cm inside diameter. The tail of the nitrogen vessel is $5.45-\mathrm{cm}$ outside diameter, the tolerance between a pole face and the glass being only $0.5 \mathrm{~mm}$. The wide part is $58 \mathrm{~cm}$ long; the outside diameter is $11.5 \mathrm{~cm}$.

The nitrogen Dewar cap is provided with monel tubes for the admission of liquid nitrogen and escape of vapor. Five monel tubes pass through the helium Dewar cap. One accommodates the transfer syphon and may be sealed off after transfer by a plug and a rubber sleeve; glass tubes pass through each of two others (sealed in by black wax) for connection between the experimental apparatus and the highvacuum system; the remaining two, which are much narrower, provide the connection to the manometer system and an inlet for the leads to the coils of the mutual-induction bridge.

The rough-vacuum pump (capacity 50 liters/sec) is located in the basement beneath the cryomagnetic laboratory. It is mounted on vibration eliminators, because even the energy of small vibrations of the cryostat is sufficient to develop an appreciable amount of heat in a demagnetized sample [8]. A vertical 5-in. copper line passes from a short length of flexible steel tubing at the inlet to the pump to the room above and is surmounted by a 3 -in. globe valve; the latter connects through an S-shaped 3-in. inside diameter reinforced flexible rubber hose to the 3 -in. copper line, which is mounted on the moving arm. The arm is then free to rotate about its supporting pillar without interruption of pumping the bath, and the vibrations of the pump are not transmitted to the cryostat. As a final step in the suppression of vibrations, a large sandbox was built around the 5 -in. line and valve in the upper room. The cryostat can be conveniently swung out of the magnet and into the framework supporting the earth's field compensation coils, which may then be raised into position (see section 6 ).

The temperature of the liqiud helium in the cryostat is derived in the conventional way from its vapor pressure. A system of three manometers was constructed: First, a mercury manometer; a millimeter scale engraved on a glass mirror was mounted directly behind the tubes of the manometer and permitted readings of pressures in the region between $1 \mathrm{~atm}$ and $5 \mathrm{~cm}$ with sufficient precision without the use of a cathetometer. For the region between 5 and $0.5 \mathrm{~cm}$ of mercury, a similar oil manometer was constructed. Lower pressures are read with the help of a small McLeod gage with a wide capillary. It is sensitive in the region between 1 and $0.01 \mathrm{~mm}$ of mercury. The whole manometer assembly is shown in figure 3 .

The high-vacuum system as a whole is mounted on the rotating arm and follows the movements of the cryostat. It consists of an oil-diffusion pump (capacity 20 liters/sec), a spherical Pyrex liquid-air trap, and a valve system. A side tube permits the admission of exchange gas to the experimental chamber directly from the cryostat (see fig. 2). Pressures in the region between 1 and $10^{-3} \mathrm{~mm}$ of $\mathrm{Hg}$ are measured by means of a Pirani-gage, and lower pressures (down to $10^{-5} \mathrm{~mm}$ ) are read with an ion gage.

When the cryostat is between the electromagnet poles or in the earth's field compensator, it is rigidly held in position by means of locking clamps on the beam, which are provided with a screw for accurate adjustment (fig. 4).

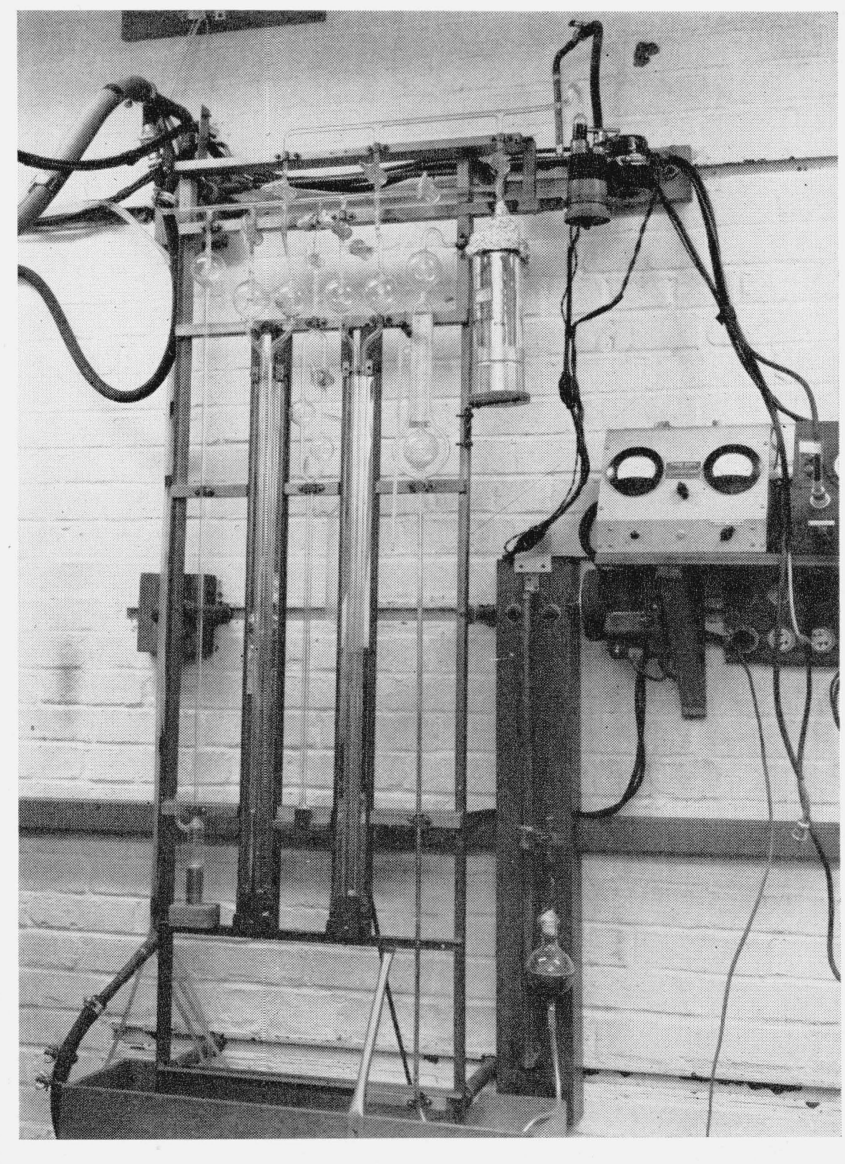

Figure 3. Manometer system.

Mounted on the framework, from left to right, are (1) over-pressure release "bubbler," (2) mercury manometer, (3) differential oil manometer, (4) oil manometer, and (5) McLeod gage. 


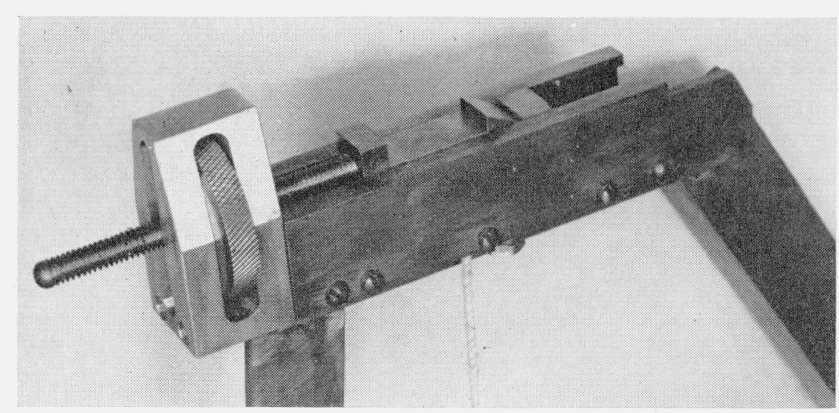

FIGURE 4. Clamping device for locking in position the rotating arm.

Two are used, one above the electromagnet and one above the earth's-field compensation coils.

\section{Experimental Chamber}

For adiabatic demagnetization experiments, in which only the temperature and magnetic properties of the salt itself are investigated, the apparatus is extremely simple. It comprises a paramagnetic salt specimen mounted within a vacuum case and thermally isolated from the walls as efficiently as possible. This may be achieved by suspending the specimen from fine nylon fibers or mounting it in an "eggcup" fixed to the lower end of the vacuum. case by a thermally insulating pedestal. The vacuum case may be made either of metal or of glass. Glass apparatus is always preferable to metal because the latter can cause undesirable electromagnetic effects in the measuring equipment through the agency of eddy currents and, on occasion, ferromagnetic impurity. A metal apparatus is more convenient, however, for repeated assembly and dismantling. In this case the tubing should be very thin-walled, and of high electric resistivity nonmagnetic material. Finally, the "cleanup" of exchange gas on pumping is markedly more rapid when glass is used rather than metal.

In this apparatus the vacuum case is made of glass. Soft glass is used to avoid the possibility of heating (and so seriously damaging) the salt during assembly. (Some paramagnetic salts lose their water of crystallization even at $25^{\circ}$ C.) The Pyrex of the high-vacuum system is joined to the soft-glass tubing emerging from the cryostat cap by means of a tapered joint (see fig. 2). If the egg-cup type of specimen support is used, the pedestal may be of thin-walled glass tubing with an extra-thin midsection.

The pumping tube emerging from the vacuum case is bent as shown in figure 2 to provide radiation traps. With the sample mounted inside the vacuum case the whole apparatus is silvered externally to a point above the traps, and a protecting coating of suitable varnish is applied. The radiationscreening precautions are of paramount importance in minimizing the heat influx into the specimen after demagnetization. The glass tube above the silvering is coated with aquadag over its whole length so that only a very small cone of roomtemperature radiation can penetrate into the first trap. The secondary coil of the mutual inductance (see below) is wound on to the tube around the specimen and covered with a wrapping of very thin paper. Care must be taken to avoid electrical leaks from the lead wires to the aquadag layer.

The paramagnetic salt may be in the form of powder or a single crystal. In order to be able to calculate the internal field of the specimen, it should be spherical or ellipsoidal. Not only is heating: dangerous for the salt, but also pumping may cause loss of water of crystallization, expecially if the sample is in powdered form. The experimental chamber must never be evacuated at room temperature; evacuation is safe, however, at temperatures well below $0^{\circ}$ C. A single-crystal specimen may be protected by a coating of varnish, collodion, etc.

\section{Earth's Field Compensation and Auxiliary Field Coils}

The necessary apparatus, which is strictly free of iron or other magnetic material, was built into one unit and is mounted in a brass-tubing framework. By means of counterweights and supporting cables over pulleys, the unit, weighing some 180-lb, can be conveniently raised and lowered to permit movement of the cryostat (see above) into and out of the field.

The earth's field compensation is effected by means of three pairs of mutually perpendicular square coils, the units of each pair being separated a distance of 0.55 times the side of the square, which is a "pseudo-Helmholtz" condition for maximum uniformity of field at the center of the unit. The windings are set in peripheral slots cut in wooden frames, and the current in each of the three pairs of coils (which are fed from a common accumulator source) is controlled through a rheostat and read on a small d-c milliammeter. The wooden frames are secured together at their points of contact by small brass right-angle brackets and wood screws. A flip-coil and ballistic galvanometer circuit is used as a detector of magnetic field, and the currents are adjusted to reduce to zero each of the three components of the field at the center.

The 500-oersted coils are wound on cylindrical wooden cores between end-plates of Bakelite. They are made in two sizes, the smaller pair just far enough apart to accommodate the tail of the cryostat and the larger pair approaching close to the walls of the cryostat where it widens. The end-plates of the larger pair are square and $3 / 4-i n$. threaded brass rods pass through holes at the corners. These rods are fitted with pulleys at each end, and these guide the assembly along the vertical members of the supporting framework. The smaller coils are bolted to the large ones, and the end-plates of each coil are held by bolts passing through the cores. These bolts must be sufficiently strong to withstand the large strains set up during the winding. In 
each pair the coils are separated by a distance equal to the mean radius of the windings to obtain a reasonably uniform field at the center. The assembly is shown in figure 5 .

The constructional details are as follows: Large coils: Each winding is 1,080 turns of No. 14 $\frac{1}{2}$ gage Formex wire, resistance 10.8 ohms; calculated fields for the two coils in parallel, 23.15 oersteds/amp; calculated initial rate of rise of temperature, 4.2 $\mathrm{deg} / \mathrm{min}$ at 6 amp. Small coils: Each winding is 588 turns of No. 16 gage Formex wire, resistance 4.7 ohms; calculated field for the two coils in parallel, 24.75 oersted/amp; calculated initial rate of rise of temperature $8.4 \mathrm{deg} / \mathrm{min}$ at $6 \mathrm{amp}$.

The coils are connected in a symmetrical seriesparallel manner and fed from a battery d-c supply, giving 52.5 oersteds/amp. In general, two sources of $10 \mathrm{v}$ and $100 \mathrm{v}$, respectively, are used, which may be selected by means of a double-pole double-throw switch. For fast measurements it is convenient to be able to vary the ineld in predetermined steps, and for this a panel was constructed to carry a network

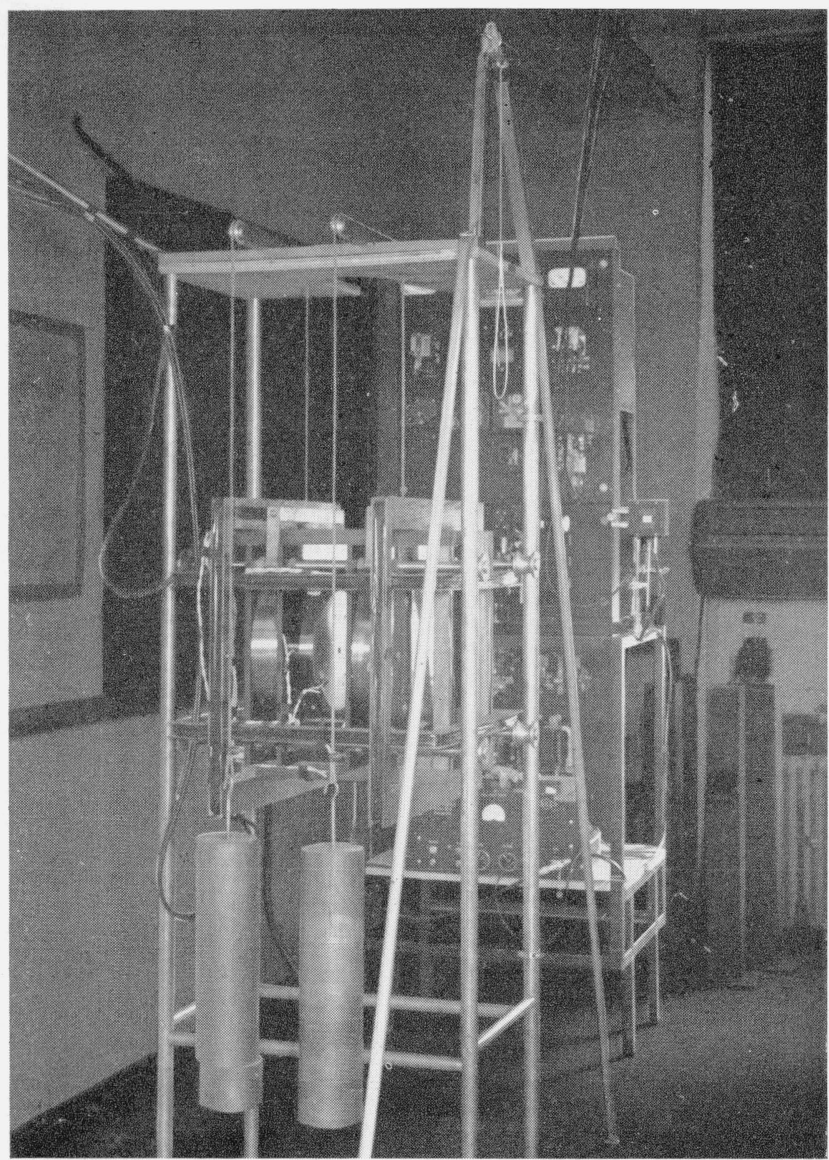

FiguRE 5. Earth's-field compensation coils and 500-oersted Helmholtz-coils unit.

The control panel for the synchronous motor of the 25-kw motor-generator set is in the background. of ohmite resistors. A rotary selector switch mounted on the front of the panel permits the cutting in or out of the resistors in succession, and a second rotary selector switch permits one to select the appropriate current range on one or other of two 3-range d-c ammeters.

To reduce contact-sparking a small thyratron is connected across the coils as shown in figure 6 , so that when the main circuit breaker is opened the energy stored in the coils may be dissipated in the circuit now closed by the thyratron instead of in a spark across the switch contacts.

The control panel is shown in ifigure 7 .

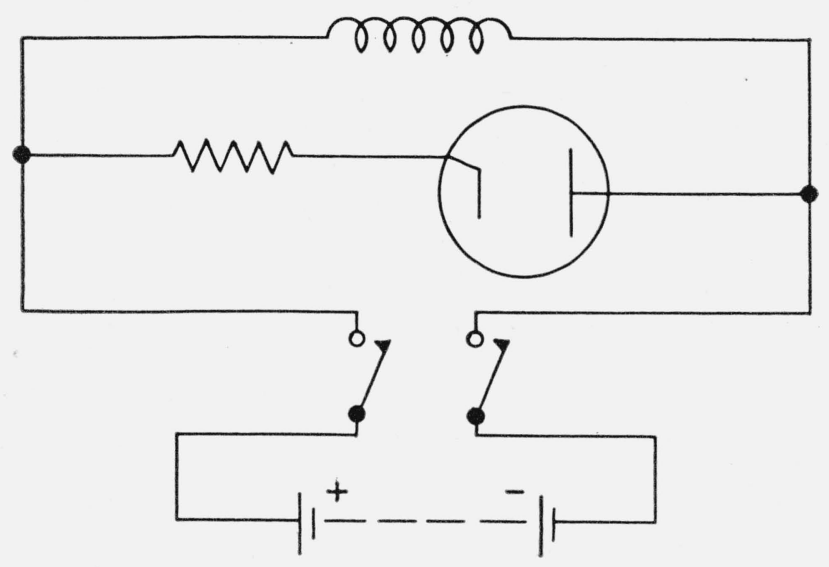

FIGURE 6. Thyratron circuit for reducing arcing at switch contacts on deenergizing 500-oersted coils.

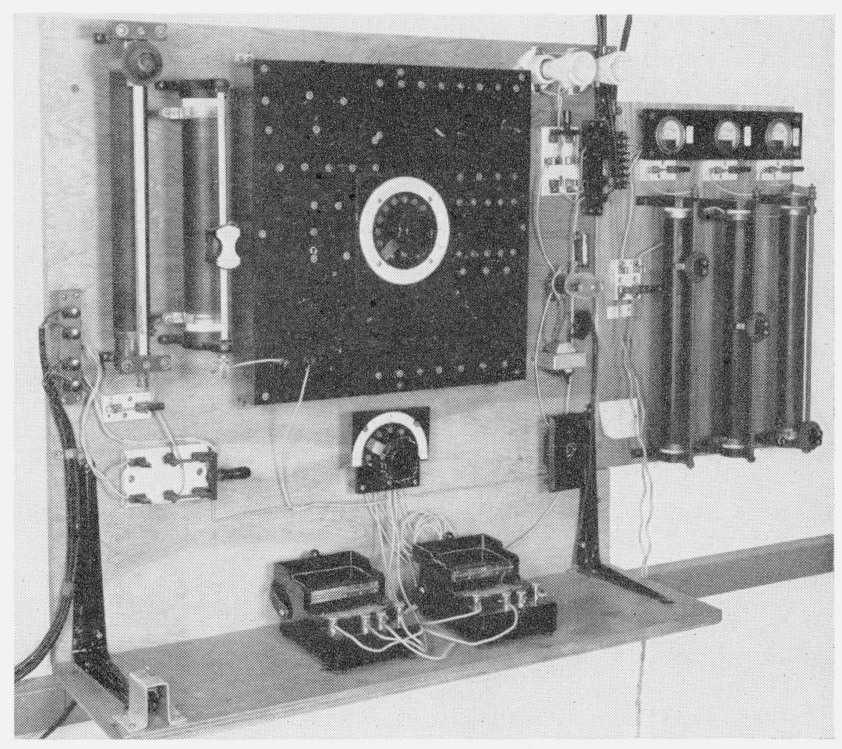

Figure 7. Auxiliary-field control unit.

The main panel contains the control circuit for the 500-oersted coils and the three rheostats for adjusting the current in each of the earth's-field compensation coil-pairs may be seen at the right. 


\section{Mutual-Inductance Bridge}

The bridge is of the Hartshorn type [9] and is a close copy of the one used for the demagnetization work in the Kamerlingh Onnes Laboratory [10]. It can be used both for ballistic and a-c measurements. As was already mentioned, the a-c method is the more convenient one: A higher precision can be obtained by it and more measurements can be taken per unit time; moreover, at the lowest temperatures, where hysteresis and relaxation phenomena occur, the bridge gives valuable information on the heat absorption by the salt from the alternating magnetic field. On the other hand, this heat may give rise to a fast warming up of the sample, and hence, when measurements are required at constant temperature, the ballistic method must be preferred in this region. With the latter, moreover, data can be obtained on the remanent magnetic moment and the shape of the hysteresis loop.

Schematic diagrams for both the a-c and the ballistic bridges are given in figure 8 , a and b. S represents the paramagnetic salt surrounded by the primary and secondary windings of a mutual induc-

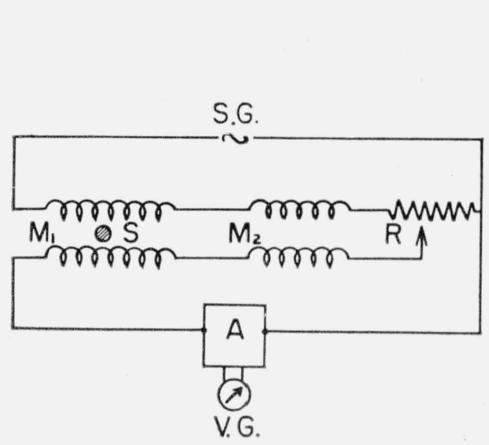

(a)

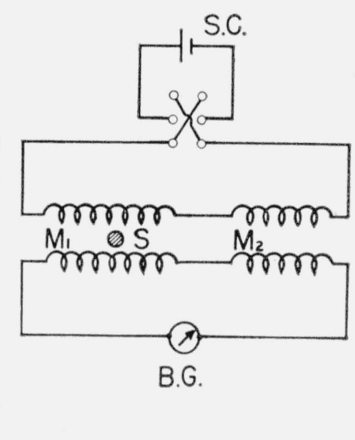

(b)
FIgURE 8. Schematic drawing of alternating. current and ballistic measuring circuits.

(a) a-c measuring circuit: $\mathrm{SG}$, signal generator; $\mathrm{S}$, paramagnetic salt; $\mathrm{M}_{1}$, cryostat mutual inductance; $\mathrm{M}_{2}$, variable mutual inductance, opposing $\mathrm{M}_{1} ; \mathrm{T}$, phase-shift potentiometer; $\mathrm{A}$, amplifier; and VG, vibration glavanometer.

(b) ballistic measuring circuit. SC, storage cell; BG, ballistic galvanometer.

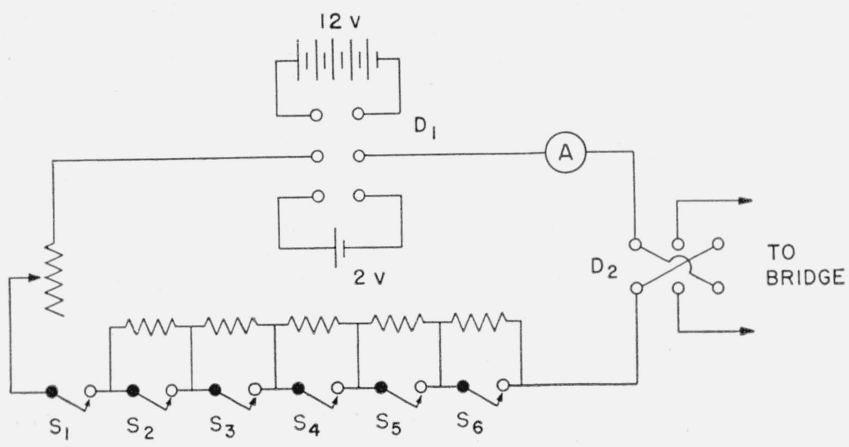

FIgURE 9. Direct-current power-supply circuit.

$D_{1}, 2$ - to 12-v selector switch; A, ammeter; $S_{1}$ to $S_{6}$, switches for step-variation of current; $\mathrm{D}_{2}$, reversing switch. tance, $\mathrm{M}_{1}$. This is connected in series with a variable mutual inductance, $\mathrm{M}_{2}$, provided by the bridge circuit. In the case of the a-c bridge the primary circuit is fed from a low-frequency (about $200 \mathrm{c} / \mathrm{s}$ ) signal generator, SG, the detecting device being a vibration galvanometer, VG, preceded by an amplifier, $\mathrm{A}$. The mutual inductance, $\mathrm{M}_{2}$, is continuously variable so that exact compensation of $\mathrm{M}_{1}$ is possible, VG being used as a null detector. If a-c losses occur in $\mathrm{S}$, these will give rise to a phase shift in the secondary voltage of $\mathrm{M}_{1}$. Now this voltage can be resolved into two components, of which only one can be compensated by $\mathrm{M}_{2}$. The other component, which is in quadrature with the voltage of $\mathrm{M}_{2}$ (and hence in phase, or $180^{\circ}$ out of phase, with the primary current) can be compensated by taking off a small voltage from a potentiometer, $R$, in the primary circuit. Zero deflection of VG can only be obtained if both $\mathrm{M}_{2}$ and $\mathrm{R}$ are set to the correct value. $^{2}$

For ballistic measurements the primary circuit is fed from a storage cell, SC, with a combination of switches and adjustable resistors. In this case only larger dials of $\mathrm{M}_{2}$ are used, and $\mathrm{M}_{1}$ is only approximately compensated by $\mathrm{M}_{2}$. The susceptibility (or the magnetic moment) of $\mathrm{S}$ is now calculated from $\mathrm{M}_{2}$ and the residual deflection of a ballistic galvanometer, BG. In this case the resistance, $\mathrm{R}$, is not needed in the circuit.

The a-c and ballistic bridges are built into one network. The transition from one to the other can be made in a few seconds by setting some switches. $\mathrm{SG}$ is then replaced by the storage cell, VG, and A by $B G$, and $R$ is switched out of the circuit.

The following are short descriptions of the separate components of the bridge network.

The a-c power supply consists of a commercial audiofrequency signal generator. The frequency can be varied between 20 and $20,000 \mathrm{c} / \mathrm{s}$, but generally frequencies of a few hundred cycles per second are used. In the present work the standard frequency is $210 \mathrm{c} / \mathrm{s}$, just between two of the mains harmonics, so that disturbing influences from the mains are as small as possible. At the rated power output of $5 \mathrm{w}$ the distortion of the signal is less than 1 percent. The current is read on a precision milliammeter, with a set of internal shunts for different ranges.

The d-c power supply is shown diagrammatically in figure 9. With the dp-dt switch, $\mathrm{D}_{1}$, either of two storage batteries (2 and $12 \mathrm{v}$ ) may be selected. Ballistic susceptibility measurements are performed by reversing the primary current through $\mathrm{M}_{1}$ and $\dot{\mathrm{M}}_{2}$ by means of $\mathrm{D}_{2}$. Measurements of hysteresis loops can be made by switching the current on or off in six approximately equal steps with the help of

2 If a-c losses of the above-mentioned kind occur, the magnetic behavior of the salt can be described by means of a complex susceptibility: $\chi=\chi^{\prime}-i \chi^{\prime \prime}$, where $\chi^{\prime}$, the "inductive component," gives rise to the part of the magnetic moment that $\chi$, the "inductive component," gives rise to the part of the magnetic moment that can be compensated by $\mathrm{M}_{2}$, and $\chi$ ", the "resistive component," can be compenfield is equal to $\frac{1}{2} h_{0}^{2} \omega \chi^{\prime \prime}[4]$ (where $h_{0}$ is the amplitude of the field), this heat can field is equal to $\frac{1}{2} h_{0}{ }^{2} \omega \chi^{\prime \prime} \mid 4$ ] (where $h_{0}$ is the amplitude of the field), this heat can
be derived at once from the value of $\mathrm{R}$. For the calculation of the magnetic be derived at once from the value of $\mathrm{R}$. For the calculation o.
moment (in magnitude and in phase) both $\mathrm{M}_{2}$ and $\mathrm{R}$ are needed.

It should be noted that spurious a-c losses in the bridge also gave rise to a contribution to $\mathrm{R}$. 
$\mathrm{S}_{1} \ldots \mathrm{S}_{6}$. If only the remanent magnetic moment is of interest, the current is made and broken by $\mathrm{S}_{1}$. This can be done in both directions by reversing $\mathrm{D}_{2}$, when $\mathrm{S}_{1}$ is open. The current is read on the precision milliammeter, $\mathrm{A}$, with a set of internal shunts for different ranges.

The primary coil in the mutual inductance, $\mathrm{M}_{1}$, consists of one layer of No. 30 double-silk covered enameled copper wire wound onto the tail of the liquid-helium Dewar and hence immersed in the liquid nitrogen (see fig. 2). The secondary coil is wound, in three sections, on the outside of the vacuum case. The center section surrounds the salt specimen and consists of several hundred turns of No. 42 Formvar-enameled copper wire (Formex) the number being the greater, the weaker is the paramagnetism of the salt and the smaller the size of the sample. The remaining two sections are wound above and below this in the opposite direction at a separation of about $1 \mathrm{~cm}$, each containing half the number of turns in the center winding. With this arrangement of coils the mutual inductance of the whole set will be zero until the salt is placed within the center winding. Thus at high temperatures where $\chi^{\prime}$ is very small, the bridge may be balanced at a low value of $\mathrm{M}_{2}$. As both the primary and secondary coils are at a low temperature, their resistance is low, and this is advantageous for the stability of the bridge.

The mutual inductance, $\mathrm{M}_{2}$, is mounted on the measuring table, of which an over-all view is given in figure 10. Different equipment is used for a-c and ballistic measurements.

The a-c part of $\mathrm{M}_{2}$ consists of three units. The main unit is variable up to $3 \mathrm{mh}$ in steps of about $3 \mu \mathrm{h}$, the variation being made by switching the turns of the secondary coil in and out of the circuit by means of a three-decade dial system. The second unit, the so-called "tenths of turns unit", consists of one decade, each setting being equivalent to one-tenth of the smallest unit of the main mutual inductance. The third unit, the "variometer" is continuously variable and is a fine adjustment on the foregoing one.

The primary coil of the main mutual inductance comprises two parallel solenoids wound in opposite directions and connected in series. They consist of one layer of No. 20 Formex wire, wound on cylindrical ceramic formers, $40 \mathrm{~cm}$ long and $4-\mathrm{cm}$ outside diameter. In order to reduce the capacity between subsequent turns, a nylon thread of $0.3-\mathrm{mm}$ thickness was wound between the turns of the copper wire. In order to prevent the accumulation of moisture between the windings, the solenoids were vacuum-impregnated with microcrystalline wax and covered with oil tape before the wax had hardened.

Bakelite bobbins fitting closely over the primary solenoids carry the secondary coil. The latter is wound from 10-strand cable made up from No. 30 double-silk covered enameled copper wire as described below. Three coils are wound on a bobbin, one having 100 turns of this cable, one having 10 turns, and one consisting of a single loop. The location of the turns is shown in figure 11; they are wound in such a way that all the coils have the same average diameter. The secondary coils are im.pregnated in the same way as the primary solenoids.

The 10-strand cable is made with the help of a sim ple device, as follows: Wires from 10 supply spools spaced around the perimeter of a large plywood disk are led radially toward the center of the disk and then through right-angled glass tubes parallel to the axle. The latter projects from the opposite face and is mounted in a lathe. As the disk is rotated the 10 wires are pulled continuously outward and the cable forms; in winding it on to a storage spool the latter must be given an occasional turn to reduce the possibility of kinking. Doubtiess, an automatic device could be devised to carry out this process somewhat more conveniently and rapidly,

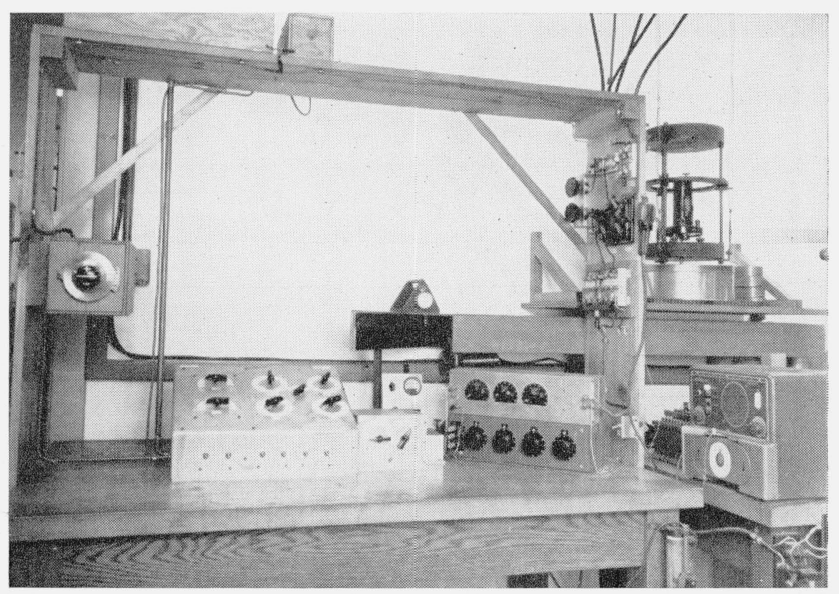

Figure 10. General view of bridge table.

From left to right, variometer, mutual-inductance dial box, sensitivity control and galvanometer selector, and resistance boxes for Peff network (see fig. 15 and text). The main mutual inductances and the "one-tenths" unit are enclosed in the two boxes at top left, and the ballistic galvanometer may be seen at top right.

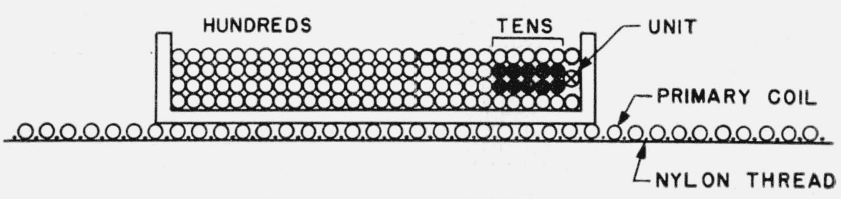

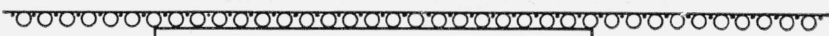

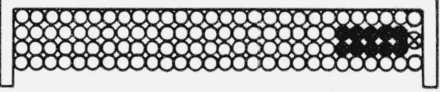

Figure 11. Mutual-inductance unit (detail) showing location of secondary windings which are made from 10-strand cable. 
but it has been found that two persons (one pulling steadily and one storing the cable) can manufacture the cable at a rate of 5 to $10 \mathrm{~m} / \mathrm{m}$.in with no difficulty.

The advantage of the use of this tenfold stranded wire in the bridge is obvious. It is the easiest way to obtain 10 coils that are identical to a high degree of precision (a few parts in $10^{4}$ ). The disadvantage, however, is that the capacitive coupling between the 10 coils is not very small, so that the bridge can only be used for investigations at relatively low frequencies. In practice, the upper limit is of the order of 500 cycles.

As mentioned above, two parallel primary solenoids wound in opposite directions and connected in series are used. They are set in a wooden box on a board $3 \mathrm{ft}$ above the table (fig. 10). Each solenoid is surrounded by a secondary bobbin, as described above, and the turns of these bobbins are also connected in series. This arrangement has the advantage of both a negligible stray field from the primaries on the measuring table and a negligible pickup in the secondary coils from spurious alternating magnetic fields. The resultant 1,110 turns (in fact, pairs of turns on the two bobbins) are led to three selector switches providing decades of hundreds, tens, and units of turns. Due to the fact that all the windings have the same average diameter, the relations $10 \times 10$ turns $=1 \times 100$ turns and $10 \times 1$ turn $=1 \times 10$ turns hold with a high degree of precision. Their validity can be impruved further by shifting the secondary bobbins along the primary coils, making use of the fact that the field of a solenoid of finite length is not exactly homogeneous. After some adjustment we obtained: $10 \times 10$ turns $-1 \times 100$ turns $=0.003$ turn, and $10 \times 1$ turn $1 \times 10$ turns $=0.013$ turn. In this position the units of turns correspond to a mutual inductance of $3.061 \mu \mathrm{h}$ each. For very precise investigations this correction may be applied, but for ordinary demagnetization work it can be neglected. The rotary selector switches (commercial low-thermalemf type, with 11 contacts) are mounted in an aluminum switchbox placed on the bridge table. The cables are led from the bobbins to the switchbox through a copper tube to minimize electrical pickup (see fig. 10).

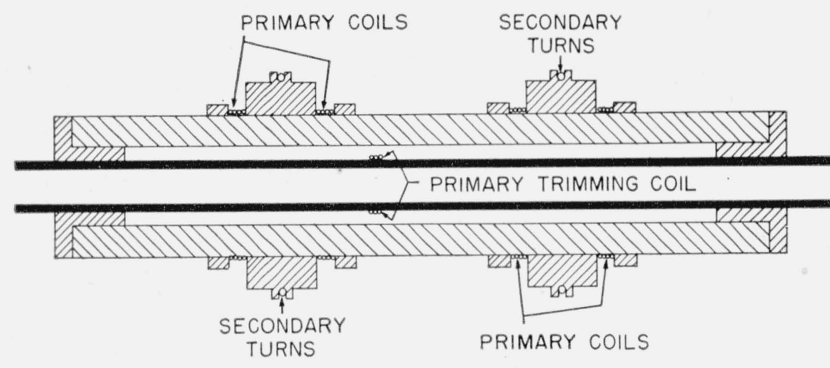

Figure 12. "One-tenths" mutual inductance (see text). The secondary winding for each half of the unit comprises a single turn of $10-$
strand cable and is located at a position of minimum primary field to ensure that each strand encompasses the same magnetic flux.
The "tenths of turns unit" is constructed in a similar manner to the above-described unit and is depicted diagrammatically in figure 12 . This unit also consists of two symmetrical parts wound in opposite directions in order to minimize stray fields and pickup effects. The primary of each part is wound in the shape of a Helmholtz coil, with three turns on each half. The secondary consists of one turn of tenfold stranded cable of such a diameter that the wires pass through a region of minimum primary field, so that the flux linkage is little affected by irregularity of winding. It is connected to a fourth rotary selector switch in the switch box on the table. A small trimming coil wound on a glass tube inside the unit and connected in series with the primary winding may be moved along the axis of symmetry in order to adjust the total mutual inductance exactly to that of one turn of the main mutual inductance.

The variometer is shown in figure 13. Again, this unit is double to minimize stray fields and pickup. The Bakelite former (11-cm diameter) holds a peripheral primary winding of 5 turns of No. 20 Formex. The secondary winding is actually a single, incomplete, loop of brass fixed to one face of the Bakelite former, with one lead soldered in the rear to the midpoint of the loop and the other connected to the sliding contact, which moves over the brass ring. The mutual inductance is positive or negative according to whether the contact is moved clockwise or counterclockwise away from the midpoint. The position of the sliding contact is indicated by a dial; the point for which the mutual inductance is equal to one-tenth of a turn of the main unit is found by experiment and marked. The space between this point and the midpoint is then graduated linearly with 100 divisions, and this is repeated for the opposite rotation.

The ballistic part of $\mathrm{M}_{2}$ is very similar to the main unit of the a-c part. Actually, the same primary solenoids are used in both cases. Another pair of

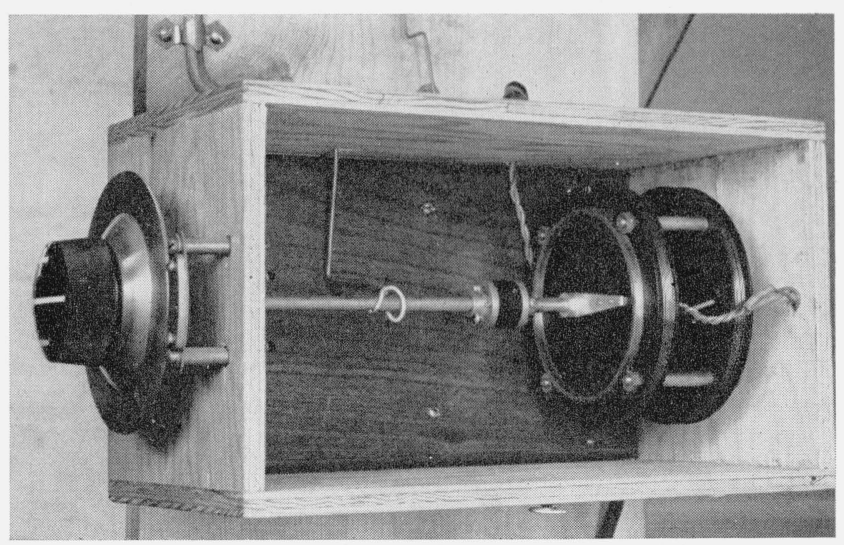

Figure 13. Variometer.

5. The two halves of the primary winding and one of the secondaries - a single (split) ring of brass - may be seen at the right. The dial (left) is graduated \pm 100 , one scale division corresponding to approximately 0.003 microhenry. 
bobbins was mounted on the primaries, and secondary coils of tenfold stranded cable were wound on them, but only the hundreds and tens of turns, since the ballistic measurements require only partial compensation of $\mathrm{M}_{1}$ by $\mathrm{M}_{2}$ (see the beginning of section 7$)$. The coils were connected to double (22 point) rotary selector switches and 20 noninductive spools of copper wire were wound, each trimmed to the resistance of a particular mutual-inductance winding. The connections were then made in such a way that as inductance turns are switched out (for instance) the appropriate noninductive resistance is switched in. By this means the total resistance of the secondary circuit remains constant, and hence the ballistic sensitivity of the galvanometer is constant. The reason that these coils are not also used for the a-c measurements is that the compensator resistances give a noticeable increase of the spurious a-c losses in the bridge circuit. An inside view of the switchbox showing the wiring to the switches and the ballistic compensator resistances is given in figure 14.

The phase-shift potentiometer may be constructed as $\mathrm{R}$ in figure 8 , a, but a practical difficulty is that resistances of the order of $10^{-4}$ and $10^{-5}$ ohm must be measured with some precision. For this reason the circuit shown in figure 15 was built, in which $r_{1}$ is a 0.1 -ohm fixed resistance, $R$ is a three-decade resistance-box variable between 100 and $10^{5} \mathrm{ohms}$, and $r_{2}$ is a four-decade box variable from 0.1 to 1,000 ohms. The voltage taken off in the secondary circuit per unit current of the primary is $\left(r_{1} r_{2}\right) /\left(r_{1}+r_{2}+R\right)$. This quantity is called the "effective resistance of the circuit," $R_{\text {eff }}$. If $R$ is set at a constant value well above $r_{1}$ and $r_{2}$ (for instance, $10^{4} \mathrm{ohms}$ ), $R_{\text {eff }}$ is practically linear in $r_{2}$, the proportionality factor being $r_{1} / \dot{R}$. The complete layout of the bridge is given in figures 16 (schematic) and 17 .

The grounding of the bridge is a point of importance. The system of figure 16 has the advantage that both the signal generator and the preamplifier of the vibration galvanometer are grounded. A reversing switch must be included in the circuit, however, so that phase shifts in both directions may be compensated with the $R_{\text {eff }}$ network. This problem was solved by connecting the reversing switch between $\mathrm{P}$ and $\mathrm{Q}$.

The a-c detecting device consists of a vibration galvanometer preceded by an amplifier. The design of the amplifier is very simple. It consists of three stages, a $6 \mathrm{AK} 5$, a $6 \mathrm{AS} 6$, and a $6 \mathrm{AQ} 5$ resistancecapacitance coupled to each other, and the last stage is coupled to the galvanometer with a transformer. No frequency-sensitive element has been inserted, and the maximum amplification is about a factor $10^{4}$. Under these circumstances, special attention must be given during the construction that no oscillations will take place. Signals down to $10^{-7} \mathrm{~V}$ must be detected, and the reason that no difficulties occur with the noise level from the first stage is that the frequency width of the galvanometer is very narrow, only a few cycles per second.

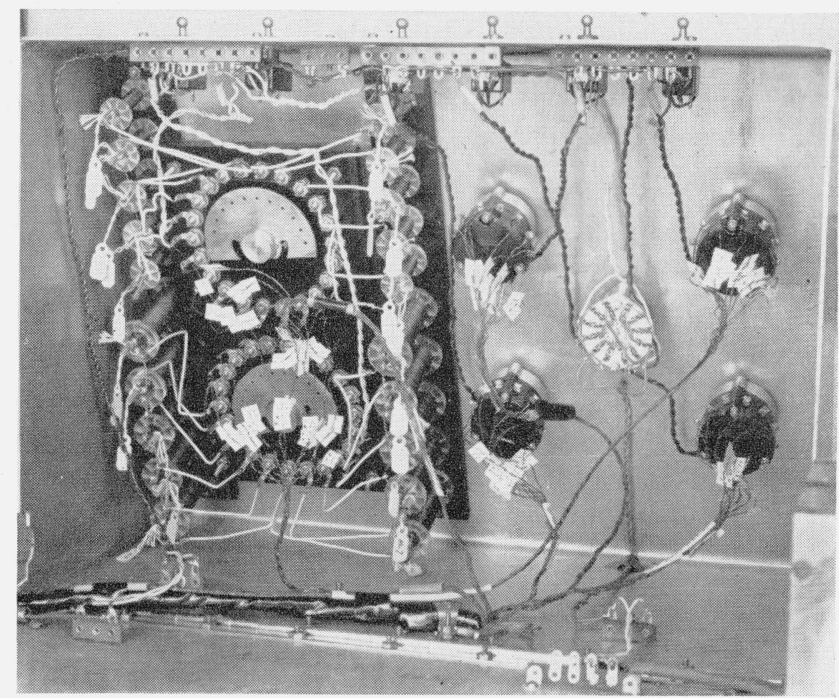

Figure 14. Interior of mutual-inductance dial box.

The two ballistic turns selectors (100-10) and 20 noninductive resistance-compensation coils are at the left; the four a-c turns selectors $(100-10-1-0.1)$ and the a-c-ballistic selector are at the right.

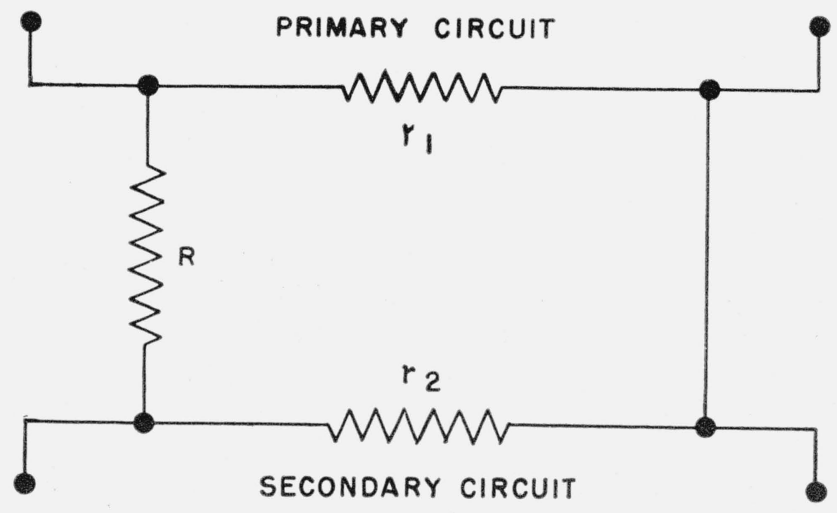

Figure 15. Phase-shift potentiometer.

$$
R_{\text {eff }}=e_{s} / i_{p}=r_{1} r_{2}\left(R+r_{1}+r_{2}\right)^{-1}
$$

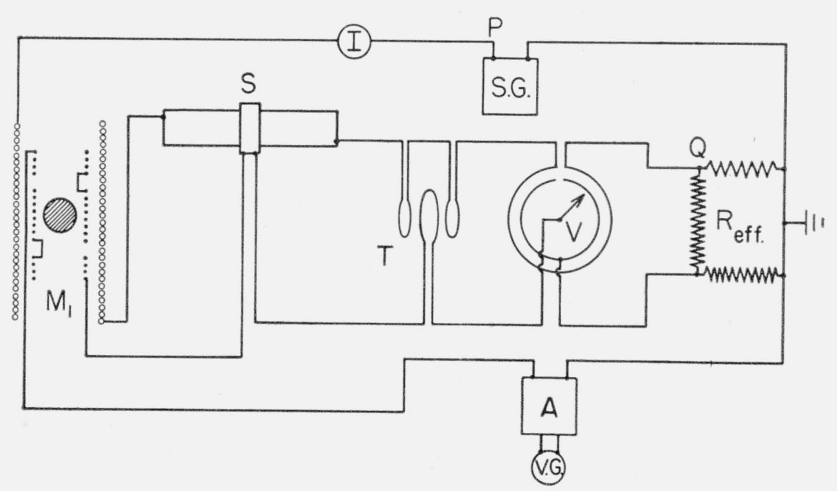

Figure 16. Mutual-inductance bridge circuit (schematic).

$\mathrm{SG}$, signal generator; I, ammeter; $\mathrm{M}_{1}$, cryostat mutual inductance; $\mathrm{S}$, main variable mutual-inductance unit; T, "one-tenths" unit; V, variometer; $P_{\text {eff }}$, phaseshift potentiometer; A, amplifier; $\mathrm{VG}$, vibration galvanometer. For $\mathrm{PQ}$, refer to text. 


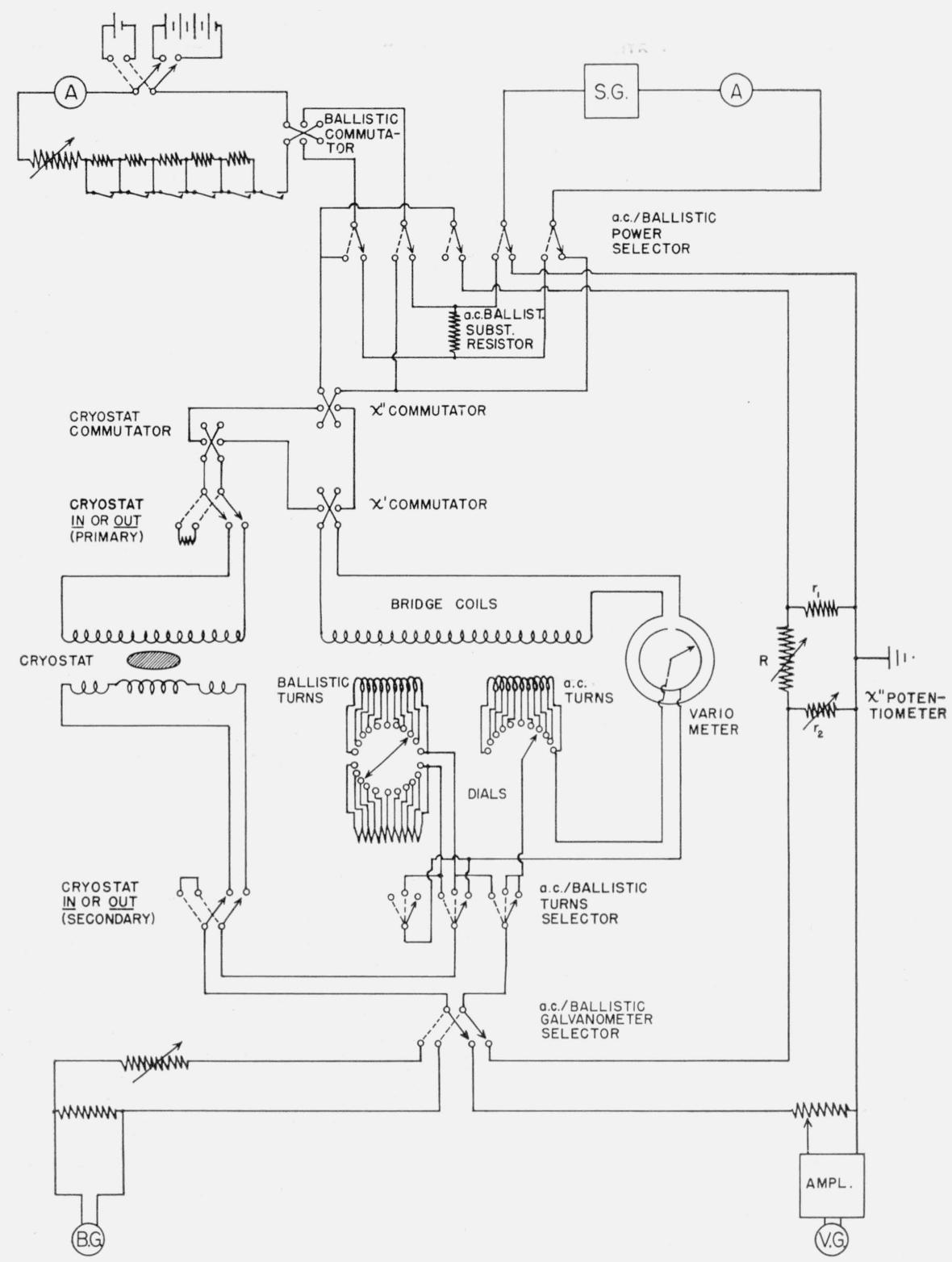

Figure 17. Circuit diagram of alternating-current/ballistic mutual-inductance bridge

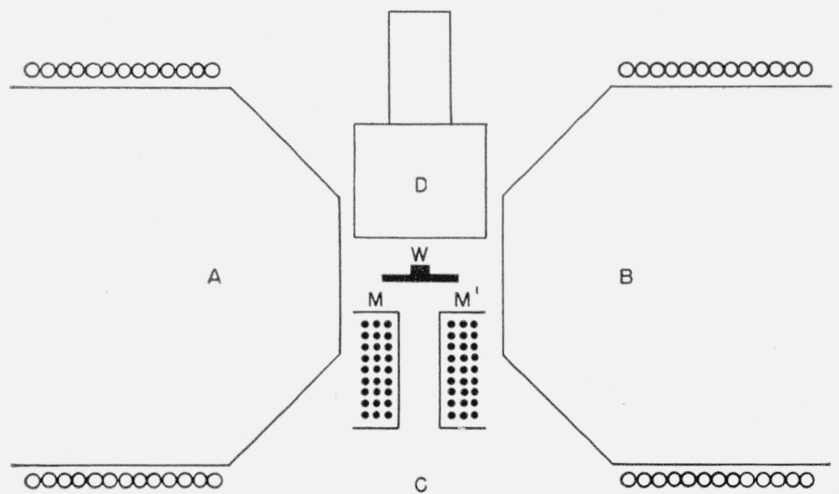

FIGURE 18. Casimir-type vibration galvanometer (horizontal cross section).

$\mathrm{AB}$, electromagnet; $\mathrm{MM}^{\prime}$, magnetic-alloy mirror; $\mathrm{W}$, phosphorbronze suspen: sion; C, deflecting coils; D, copper damping block. 
The vibration galvanometer was originally designed by Casimir and constructed in the Kamerlingh Onnes Laboratory. A cross section is shown in figure 18. The vibrating system consists of a small piece of highly magnetic alloy, MM', $2 \mathrm{~mm}$ square and $0.2 \mathrm{~mm}$ thick attached to a flat strip of phosphorbronze, W. MM' is magnetized perpendicular to the strip, and one side is ground to a mirror. The a-c coil, $\mathrm{C}$, is mounted in front. The resonance of MM' with the a-c frequency is adjusted by applying a constant d-c magnetic field, the current through electromagnet $\mathrm{AB}$ being adjusted to such a value that the deflection of $\mathrm{MM}^{\prime}$ is maximum for a given a-c current through $\mathrm{C}$. In this way the galvanometer can be tuned to any frequency between 100 and 500 c/s. D is a movable copper block, which is used for damping the vibrating system. If $\mathrm{D}$ is far away from the mirror, the damping is very small; it takes a long time for $\mathrm{MM}^{\prime}$ to reach its final amplitude for a given current through $\mathrm{C}$, and the frequency band width is so narrow that the resonance adjustment can hardly be found. If $\mathrm{D}$ is placed very close to MM', the mirror changes from one amplitude to another in a fraction of a second, the band width is still a few cycles per second, and the sensitivity of the galvanometer is quite satisfactory. If the galvanometer is in resonance and well-damped, the sensitivity is about $1 \mathrm{mv}$ in the neighborhood of 200 $\mathrm{c} / \mathrm{s}$; hence, in combination with the amplifier, signals of the order of $10^{-7} \mathrm{v}$ can be detected.

The ballistic detector device is a voltage-sensitive galvanometer with a sensitivity of $10 \mathrm{~mm} / \mu \mathrm{v}$ and a period of $5.1 \mathrm{sec}$. It is aperiodically damped with a shunt resistance, and the deflections are read by means of a telescope and a lighted scale. The commercial "ballistic galvanometers" with periods of half a minute or more are not suitable for these investigations as their ballistic sensitivity is smaller by about a factor 10 , and the long period prevents one from taking more than one reading a minute. Measurements with a fast galvanometer require some experience, but if the deflections are kept under $10 \mathrm{~cm}$ (by proper adjustment of $\mathrm{M}_{2}$ ) tenths of millimeters can be estimated easily. With the above arrangement, it is possible to take at least six readings a minute.
The possibility was considered of using a still faster galvanometer and registering the deflections photographically. This system has been applied successfully in Leiden, using galvanometers with periods of 1.3 and 0.2 sec. The method works quite satisfactorily, but in some cases difficulties occur in the interpretation of the results when the relaxation time of the salt becomes of the same order of magnitude as the swinging time of the galvanometer.

The first author, who was at the National Bureau of Standards for 1 year as a consultant, expresses his gratitude to members of the staff of the Bureau for the ready cooperation and assistance he received during his stay, for the many valuable discussions, and for the high priority that was often awarded to his work.

\section{References}

[1] P. Debye, Ann. Phys. 81, 1154 (1926); W. F. Giauque, J. Am. Chem. Soc. 49, 1864 (1926).

[2] W. F. Giauque and D. P. MeDougall, Phys. Rer. 43, 768 (1933); W. J. de Haas, E. C. Wiersma, and H. A. Kramers, Physies 1, 1 (1933).

[3] N. Kurti and F. E. Simon, Proc. Roy. Soc. 149, 152 (1935); M. H. Hebb and E. M. Purcell, J. Chem. Phys. 5, 338 (1937); H. B. G. Casimir, Magnetism and very low temperatures, Cambridge Physical Tracts (1940).

[4] D. de Klerk, Science 116, 335 (1952); D. de Klerk, Phys. Today 6, ii, p. 4-9 (February 1953).

[5] H. B. G. Casimir, D. Bijl, and F. K. du Pré, Physica 8, 449 (1941); D. Bijl, thesis, Leiden, p. 97 (1950); W. F. Giauque, J. J. Fritz, and D. N. Lyon, J. Am. Chem. Soc. $\boldsymbol{y 1}, 1657$ (1949).

[6] F. Bitter and F. E. Reed, Rev. Sci. Instr. 22, 171 (1951).

[7] M. E. Packard, Rev. Sci. Instr. 19, 435 (1948); H. S. Sommers, P. R. Weiss, and W. Halpern, Rev. Sci. Instr. 20, 244 (1949) and 22, 612 (1951).

[8] R. A. Hull, K. R. Wilkinson, and J. Wilks, Proc. Phys. Soc. [A] 64, 379 (1951).

[9] L. Hartshorn, J. Sci. Instr. 2, 145 (1925).

[10] H. B. G. Casimir, W. J. de Haas, and D. de Klerk, Physica 6, 241 (1939); W. J. de Haas and F. K. du Pré, Physica 6, 705 (1939); D. de Klerk, thesis, Leiden, p. 36-43 (1948); D. de Klerk, M. J. Steenland, and C. J. Gorter, Physica 15, 649 (1949); M. J. Steenland, thesis, Leiden, p. 12-23 (1952).

Wash Ington, April 19, 1954. 